\title{
Implementation of Picture key Algorithm in Distributed Networking to Enhance security
}

Authors

\section{Priyanka $^{1}$, Swati Gupta ${ }^{2}$}

${ }^{1}$ M.Tech Rayat Bahra Royal Institute of Management and Technology Chidana

${ }^{2}$ Asst.Prof. Rayat Bahra Royal Institute of Management and Technology Chidana

\begin{abstract}
:
In this research we have make Implementation of Picture key Algorithm in Distributed Networking to Enhance security. One popular trend is client/server computing. This is the principle that a client computer can provide certain capabilities for a user and request others from other computers that provide services for the clients. (The Web's Hypertext Transfer Protocol is an example of this idea.)Enterprises that have grown in scale over the years and those that are continuing to grow are finding it extremely challenging to manage their distributed network in the traditional client/server computing model. The recent developments in the field of cloud computing has opened up new possibilities. Cloud-based networking vendors have started to sprout offering solutions for enterprise distributed networking needs. Whether it turns out to revolutionize the distributed networking space or turns out to be another craze remains to be seen.
\end{abstract}

\section{INTRDOUCTION}

Distributed Networking is a distributed computing network system, said to be "distributed" when the computer programming and the data to be worked on are spread out over more than one computer. Usually, this is implemented over a network.

Prior to the emergence of low-cost desktop computer power, computing was generally centralized to one computer. Although such centers still exist, distribution networking applications and data operate more efficiently over a mix of desktop workstations, local area network servers, regional servers, Web servers, and other servers.

Distributed computing is a field of computer science that studies distributed systems.

\section{NEED OF RESEARCH}

Distributed Network security is sometimes more than what people always thought it to be,malware, virus, Trojan, hackers. Distributed Network security could be caused by unintentional human error and it could be compromised by human nature as well.

A common Distributed network security problem (Employees) most organizations are facing sometimes has to do with the company's employees and their various errors they make. According to Dr. Michael E. Whitman, CISM, CISSP, and the author of the textbook "Principals of Information Security, "Humans make mistakes; sometimes that is due to inexperience or improper training, and sometimes it because an incorrect assumption was reached. But regardless of the reason - and the lack of malicious intentsomething as simple as a keyboarding error has the potential to cause a worldwide Internet outage". (Whitman and Mattord 2012) The problem of piracy is another common network problem

\section{SECURITY OF DISTRIBUTED NETWORK}

During this time when the Internet provides essential communication between tens of millions of people and is being increasingly used as a tool 
for commerce, security becomes a tremendously important issue to deal with. There are many aspects to security and many applications, ranging from secure commerce and payments to private communications and protecting passwords. One essential aspect for secure communications is that of cryptography. While cryptography is necessary for secure communications, it is not sufficient. Modern cryptography intersects the disciplines of mathematics, computer science, and electrical engineering. Applications of cryptography include ATM cards, computer passwords, and electronic commerce. Cryptography prior to the modern age was effectively synonymous with encryption, the conversion of information from a readable state to apparent nonsense. The originator of an encrypted message shared the decoding technique needed to recover the original information only with intended recipients, thereby precluding unwanted persons to do the same. Since World War I and the advent of the computer, the methods used to carry out cryptology have become increasingly complex and its application more widespread.

\section{IMPLEMENTATION}

Sender side:

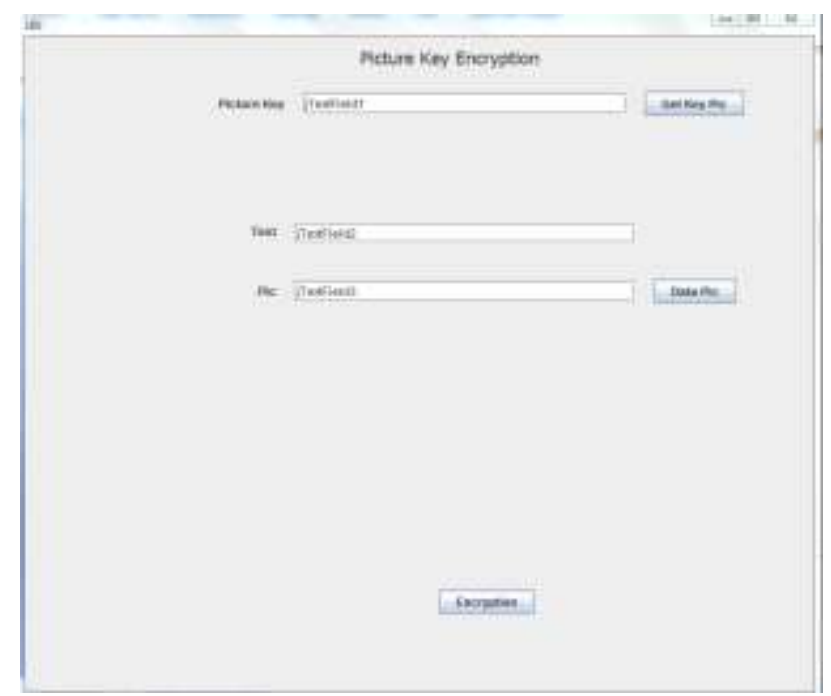

Figure 1 Snapshot of Picture Key Crptosystem For the proposed protocol key picture and data picture are shared between sender and reciver.

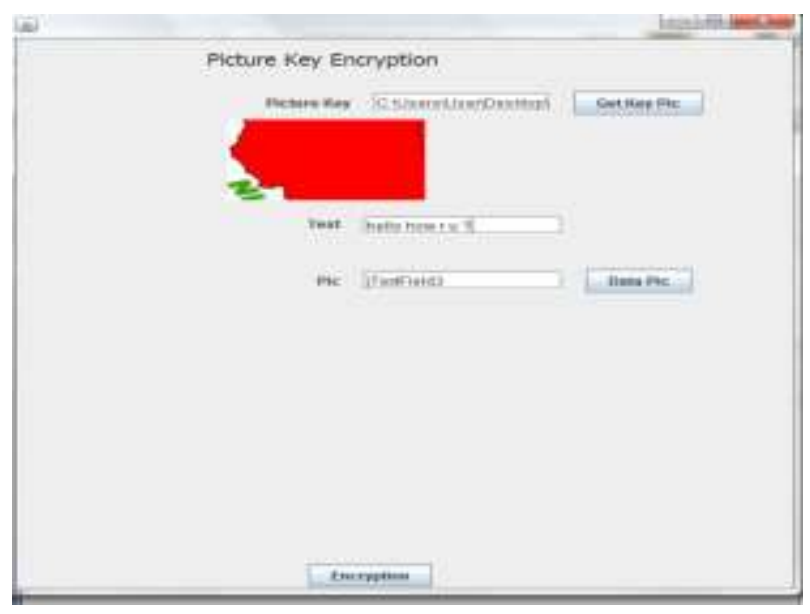

Figure 2 Snapshot of Message Encrypted with Shared Picture

In above figure 5.7 we have taken the same message "hell how $\mathrm{r}$ u?" as our plain text. The plain text is encryptred using Key picture with DES.

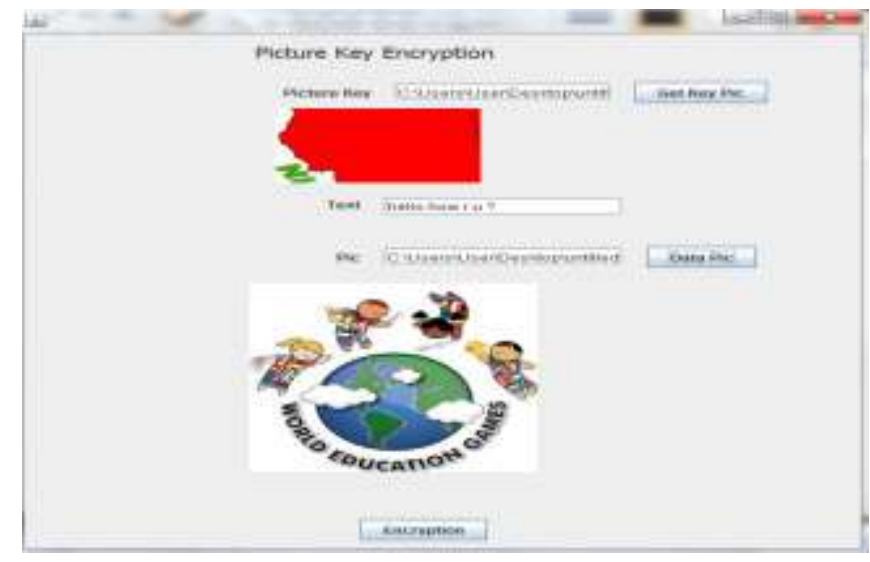

Figure 3 Snapshot of Browsing of Cover Picture for Encryption

In above figure 5.9 we have taken data picture to encrypt the cipher picture.

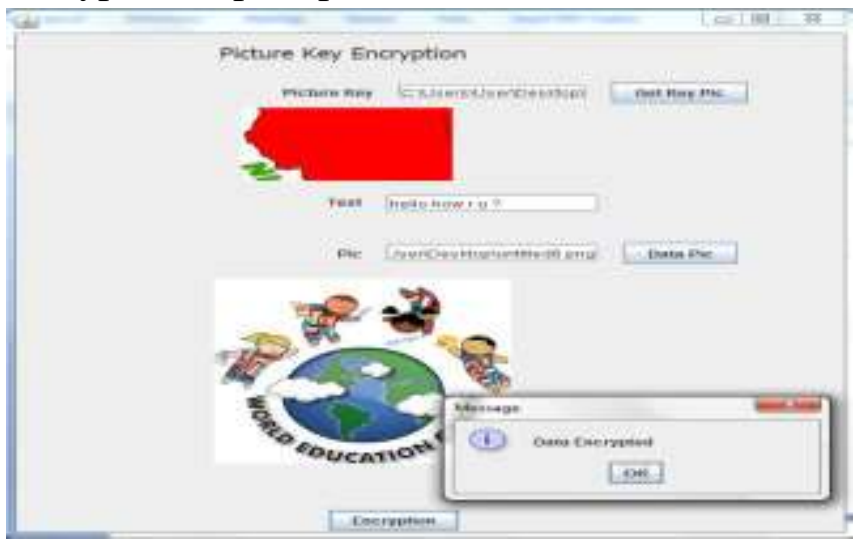

Figure 4 Snapshot Of Encrypted Message Is Again Encrypted With Cover Picture

In the above figure 5.10 the cipher picture is encrypted using the Data picture with the help of 
DES. We will get the resuktant picture. This resultant picture will be sent to receiver.

Receiver side:

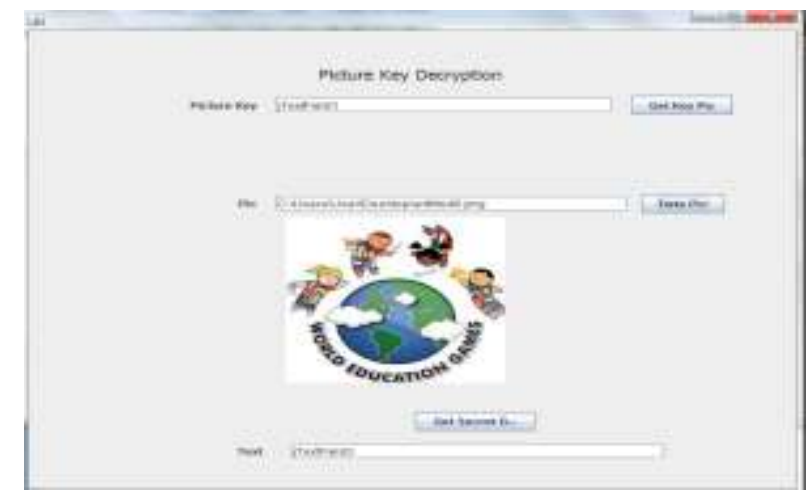

Figure 5 Snapshot of Picture Key Decryption

In the above figure 5.11 same Data picture is taken for decryption process and decryption is performed to get the cipher picture.

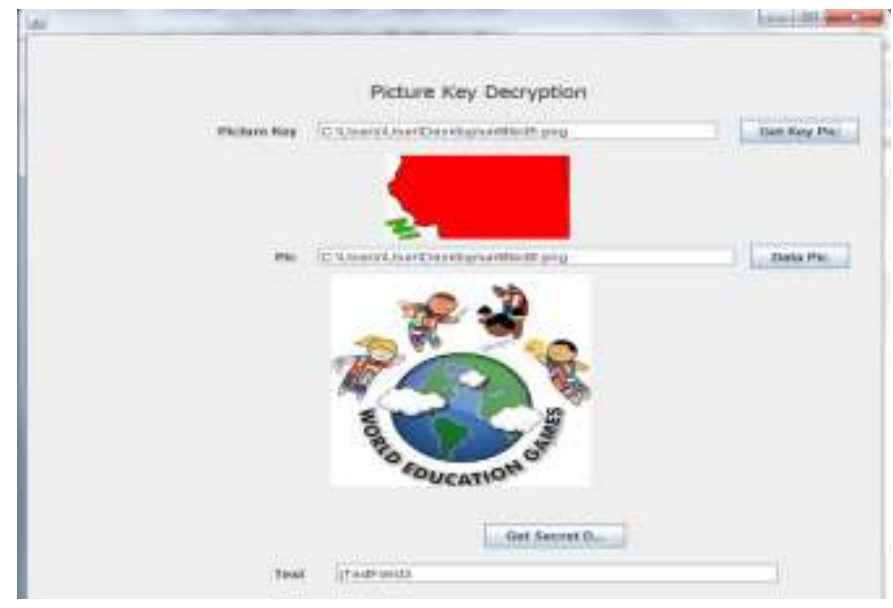

Figure 6 Snapshot Of Decryption Of Message With Cover Picture

In the above figure 5.12 the same Key picture is used for perfoming the decryption on cipher picture to get plain text.

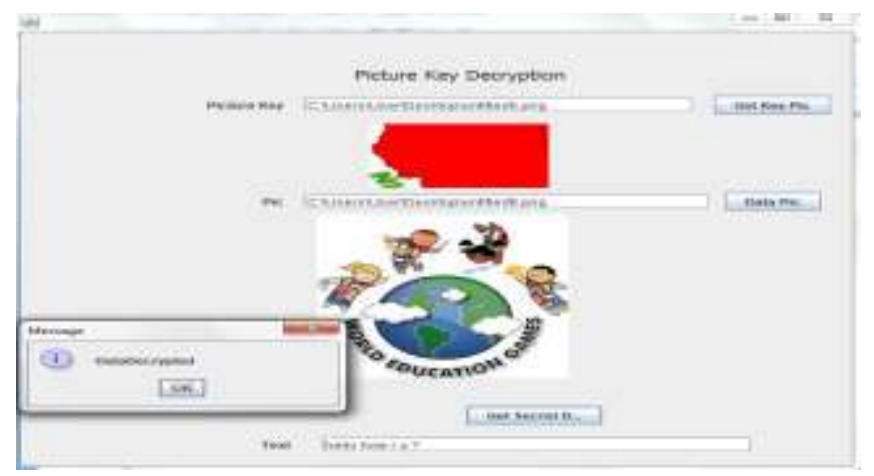

Figure 7 Snapshot Of Decryption Of Message With Shared Picture

\section{FUTURE SCOPE}

From all the available distributed and centralized systems, four most commonly used distributed systems were discussed in depth and then the security issues faced by these systems and the solutions proposed by various researchers were discussed in depth. Finally the security issues and solutions proposed for different systems were summarized and compared with each other. In future the authors are keen to develop the new and competitive approaches fir the development of secure distributed systems.

This proposed algorithm does not give complete solution of key agreement or we can say the problem of key agreement is not fully solved. In this dissertation, two new users cannot communicate electronically. Some of the existing protocol solve the problem but not fully satisfactory. But here we have defined our own ports for server and client and defined new rules for encryption and decryption this will definitely improve the security mechanism in Cloud computing environment.

\section{REFERENCE}

1. David Pointcheval, Olivier Blazy, "New Smooth Projective Hash Functions and One-Round Authenticated Key Exchange"(18_22 august 2013, Santa Barbara, California, USA), SpringerVerlag, LNCS 8042, pages 449_475.

2. David Pointcheval, Olivier Blazy, "Effcient UC-Secure Authenticated KeyExchange for Algebraic Languages"(26 February - 1 March 2013, Nara, Japan)), 16th International Conference on Practice and Theory in Public-Key Cryptography (PKC '13)Springer-Verlag, Kaoru Kurosawa Ed., Springer-Verlag.

3. David Pointcheval, "Password-based Authenticated Key Exchange" (21-23 may 2012, Darmstadt, Germany)SpringerVerlag, LNCS 7293, pages 390-397. 
4. David Pointcheval, Michel Abdalla, "Contributory Password-Authenticated Group Key Exchange with Join Capability", (February 14-18, 2011, San Francisco, CA, USA), A. Kiayias Ed. Springer-Verlag, LNCS 6558, pages 142160.

5. David Pointcheval, Xavier Boyen, "Strong Cryptography from Weak Secrets", (3 - 6 may 2010, Stellenbosch, South Africa), D. Bernstein and T. Lange Eds., SpringerVerlag, LNCS 6055, pages 297-315.

6. David Pointcheval, Michel Abdalla, "Flexible Group Key Exchange with OnDemand Computation of Subgroup Keys", (3-6 May 2010, Stellenbosch, South Africa)), D. Bernstein and T. Lange Eds., Springer-Verlag, LNCS 6055, pages 351368.

7. David Pointcheval, Michel Abdalla, "Distributed Public-Key Cryptography from Weak Secrets", (18_20 march 2009, Irvine, CA, USA), S. Jarecki and G. Tsudik Eds. Springer-Verlag, LNCS 5443, pages 139_159.

8. David Pointcheval, Michel Abdalla, "Password-Authenticated Group Key Agreement with Adaptive Security and Contributiveness", (21 - 25 june 2009, Gammarth, Tunisia) B. Preneel Ed., Springer-Verlag, LNCS 5580, pages 254271.

9. Rafael Álvarez, Leandro Tortosa, "Analysis and design of a secure key exchange scheme, Information Sciences",179 (2009), Elsevier

10. David Pointcheval, Michel Abdalla, "Anonymous and Transparent Gatewaybased Password-Authenticated Key Exchange" , December 2-4, 2008, HongKong, China - M. Franklin, L. Hui and D. Wong Eds. Springer-Verlag, LNCS 5339, pages 133-148.
11. Emmanuel Bresson, Olivier Chevassut, and David Pointcheval, "Provably-Secure Authenticated Group Diffie-Hellman Key Exchange", "ACM Transactions on Information and System Security", Vol. 10, No. 3. August 2007.

12. Kumar Mangipudi, Rajendra Katti, "A Secure Identification and Key agreement protocol with user Anonymity(SIKA)", journal homepage: www.elsevier.com/locate/cose, Computers $\&$ security 25(2006) $420-425$.

13. Chin-Chen Chang, Jung-San Lee, "An anonymous voting mechanism based on the key exchange protocol", journal homepage: www.elsevier.com/locate/cose, Computers \& security 25( 2006) 307-314. 